\title{
Promoting the Peer-Related Social Development of Young Children With Mild Developmental Delays: Effectiveness of a Comprehensive Intervention
}

\author{
Michael J. Guralnick, Robert T. Connor, Brian Neville, and Mary A. Hammond \\ Center on Human Development and Disability, University of Washington, Seattle
}

\begin{abstract}
To address the unusual peer-related social competence difficulties characteristic of young children with mild developmental delays, we conducted a randomized clinical trial to evaluate the effectiveness of a comprehensive, developmentally oriented, highly individualized intervention extending over a 2-year period. Outcome measures emphasized generalization of peer interactions in unfamiliar playgroups. Results revealed modest effects of the intervention, with children who had lower cognitive levels benefiting most. Intervention effects were best conceptualized as preventative, minimizing the negative features and atypical patterns of children's social play with peers. Our discussion of future work was focused on alternative implementation models to enhance intervention intensity, inclusion of specific subgroups of children, and direct measurement of children engaging in social tasks.
\end{abstract}

Extensive observations of preschool-age children with mild developmental (cognitive) delays in settings involving peers have revealed that substantial difficulties exist in their ability to establish social relationships with other children (Guralnick, 1999a). Comparisons to chronological age (CA) matched groups of typically developing children interacting in unstructured settings, such as playgroups, have indicated that virtually all aspects of the peer interactions of children with mild delays are affected. Specifically, in these comparisons, these children exhibit lower levels of socially interactive (maintained) play, engage in higher levels of solitary play, are less effective in gaining positive responses to their social bids, initiate fewer directive interactions, exhibit peer-interaction patterns easily disrupted by changes in playmates or other circumstances, and are less successful entering peer groups during play (Guralnick, Connor, Hammond, Gottman, \& Kinnish, 1996a; Guralnick \& Groom, 1987a, 1987b; Guralnick \& Weinhouse, 1984; Kopp, Baker, \&
Brown, 1992; Wilson, 1999). In addition, children with mild delays exhibit inappropriate problemsolving patterns during conflicts with peers, are less accepted as reflected by both peer sociometric and behavioral measures, have more difficulties forming in-depth relationships with peers as indicated by more limited linkages between peers across school and community settings, and have fewer reciprocal friendships (Guralnick, 1997; Guralnick, Gottman, \& Hammond, 1996; Guralnick \& Groom, 1987b, 1988; Guralnick et al., 1996a; Guralnick \& Paul-Brown, 1989; Guralnick et al., 1998). Related studies of school-age children reveal that these patterns persist over time, often leading to social isolation (Howell, HauserCram, \& Warfield, 2001; Taylor, Asher, \& Williams, 1987; Williams \& Asher, 1992). Of importance, the majority of these and related patterns are evident even when comparisons are made controlling for children's developmental level (see Guralnick, 1999b). Taken together, it appears that these difficulties in peer relationships are associ- 
ated with characteristics directly related to children's developmental status (i.e., mild developmental delay), are persistent and pervasive, and represent substantial problems in peer-related social competence (Guralnick, 1999a).

Contemporary conceptual models in the area of children's peer-related social competence have emphasized both children's social-information and emotion-regulation processes as well as the importance of considering peer competence within a broader developmental and ecological framework (Bierman, 2004; Denman et al., 2003; Dodge, 1991; Lemerise \& Arsenio, 2000). In addition to specific child characteristics, such as emotional reactivity (Rubin, Coplan, Fox, \& Calkins, 1995), the influence of numerous family factors on children's peer competence has been wellestablished. These include the role of parent-child interactions, direct parent actions involving coaching or arranging play activities, and parent attitudes toward peer relationships and friendships (e.g., Ladd \& Pettit, 2002). Moreover, these models have been adapted for and are highly relevant to our understanding of children with developmental delays. In particular, a number of child characteristics and family factors have been identified that contribute to those social-information and emotion-regulation difficulties that independently or jointly lead many children with delays to select ineffective or inappropriate social strategies when engaged in social tasks, such as peer group entry or resolving conflicts with peers (Guralnick, 1999a; Guralnick, Neville, Connor, \& Hammond, 2003). For example, special problems for children with delays have been identified with respect to emotion regulation, as reflected in the increased prevalence of behavior problems and the relative absence of emotion-regulation strategies in various situations (Baker, Blacher, Crnic, \& Edelbrock, 2002; Guralnick \& Groom, 1990; Keogh, Bernheimer, Haney, \& Daley, 1989; Wilson, 1999). The ability to track complex and rapidly changing social stimuli in the peer context is also compromised by extensive problems in information-processing that include attentional mechanisms (Tomporowski \& Tinsley, 1997) and working memory (Bray, Fletcher, \& Turner, 1997). Similarly, numerous family circumstances, such as social support and stress as well as aspects of parent-child relationships involving children with delays, can be adversely affected and are relevant to children's peer competence (see Guralnick,
2006, Guralnick \& Neville, 1997, and Spiker, Boyce, \& Boyce, 2002, for reviews).

Yet, despite these emerging conceptual frameworks and an increased recognition of the unusual difficulties in peer relationships that exist for a substantial proportion of children with mild developmental delays, interventions conducted for this population have generally been narrow in scope, with little consideration of the broader developmental and ecological context. The most intensive efforts have utilized teacher- and peer-mediated interventions targeting specific social behaviors in educational settings but infrequently tied to individual child profiles. Behaviorally oriented techniques have predominated, involving various types of prompting, modeling, and reinforcement of appropriate social strategies applied in both individual and small group settings. Promising immediate effects have been observed, but long-term or generalized outcomes have not been consistently established (for a review, see McEvoy, Odom, \& McConnell, 1992). Even general social skills curricula or combinations of various techniques have yielded limited or inconsistent results (Jenkins, Odom, \& Speltz, 1989; Odom et al., 1999). These problems are also evident in results of interventions for school-age children with mild disabilities (Gresham, Sugai, \& Horner, 2001).

In this study, we present the results of a randomized, prospective, controlled trial designed to address the peer-related social interaction difficulties of young children with mild developmental delays. As will be seen, the intervention approach taken here contains a number of important features not included in previous work. First, the intervention is comprehensive, seeking to influence children in both school and home settings involving both teachers and mothers in a coordinated fashion. This comprehensive approach, therefore, represents an awareness of the broader ecological influences on children's peer-related social development as well as a means for increasing the intensity of the intervention (see Guralnick, 1998, 2001). Second, the intervention is longterm, extending across a 2 -year period. This feature reflects the complexity of the issues involved, both conceptual (in terms of the developmental mechanisms that need to be considered) as well as practical (in terms of implementation). Third, in contrast to most previous work with this population, the intervention approach maintains a consistent developmental orientation. Assessment and intervention protocols follow contemporary 
developmental thinking regarding the organization of and influences on children's peer-related social competence as discussed above. Fourth, interventions are carefully individualized within this framework; linked to individual assessments and the child's profile with respect to social tasks, social strategies, social-information, and emotionregulation processes; and related developmental patterns. Similar individualized assessments establish family profiles of relevance to children's peerrelated social competence outlined earlier (e.g., parent-child interactions) and form the basis for interventions grounded in a well-integrated conceptual model. Fifth, the engagement of teachers and mothers in the intervention process is given special consideration in view of the often overwhelming demands created by circumstances associated with vulnerable children (see Berlin, O'Neal, \& Brooks-Gunn, 1998). The fact that available evidence indicates that peer-related social development of young children is given a low priority by teachers (McConnell, McEvoy, \& Odom, 1992; Michnowicz, McConnell, Peterson, \& Odom, 1995) was of particular concern. Moreover, both mothers of children with delays (Booth, 1999) and general early childhood education teachers (File, 1994) hold primarily internal rationales (attitudes) with respect to the forces governing children's peer-related social development, and mothers and teachers endorse or utilize only limited proactive socialization strategies to promote their child's peer competence (File, 1994; Guralnick et al., 2003; Odom, McConnell, \& Chandler, 1993). Consequently, we made an effort to forge a close partnership with teachers and mothers by utilizing a consultant model. Sixth, the study design permits an analysis of the impact of level of child cognitive functioning on intervention outcomes. In particular, moderator analyses were employed to determine whether children whose IQs fell at 70 or below (approximately two $S D$ s below the mean; the standard cut-off for defining mental retardation along with measures of adaptive behavior-American Psychiatric Association, 1994) were differentially responsive to the intervention in comparison to children functioning at higher cognitive levels (IQs of 71 to 90). Some evidence suggests that more vulnerable children are likely to be more responsive to comprehensive interventions (Bryant \& Maxwell, 1997; National Institute of Child and Human Development Early Child Care Research Network \& Duncan, 2003). Moreover, more children at high- er cognitive levels are less impaired in terms of peer competence at this age, thereby minimizing the potential impact of an intervention (Guralnick, Connor, Hammond, Gottman, \& Kinnish, 1996b). Finally, the evaluation methodology is designed specifically to test the effects of the comprehensive intervention in situations that require generalization of social skills to new and challenging situations. As noted, generalization has been difficult to achieve, yet it constitutes the essence of peer-related social competence.

\section{Method}

\section{Overview of Approach}

A total of seven cohorts (one per year) ranging from 11 to 17 children each year and their families were recruited to achieve a final sample of 90 participants who completed the study. Children and their families were randomly assigned (see below for details and discussion of attrition) to an intervention lasting 2 years or to a control condition. For all children, pretesting took place during the summer or early fall, and posttesting took place in the summer 2 years later. Each potential participant was informed of the possibility that they and their child might not be included in the comprehensive intervention program.

For the intervention group, a comprehensive, developmentally oriented, and coordinated school- and family-based program was implemented over the 2-year period. Control children received standard community services but maintained periodic contact with project staff. All measures were administered at both pre- and posttesting periods for children and parents in both intervention and control groups. None of the testers or coders were aware of a child's group assignment.

\section{Participants}

Young children with mild developmental delays were recruited through contact with local school districts in a large metropolitan community. Participating school districts distributed announcements describing an opportunity to participate in a research project intended to promote children's peer relations and friendships. Information was sent to all parents whose children had an Individualized Education Program (IEP) and who attended an inclusive (mainstreamed) preschool or kindergarten. Parents who were interest- 
ed in participating contacted project staff directly, who then initiated a screening and identification process. To be included in the sample, a child had to meet the following criteria: (a) be between 48 and 78 months of age, (b) have a current IEP, (c) be experiencing difficulties in peer-related social competence as expressed by parent concerns during a structured phone interview, (d) have a primary female caregiver (minimum of a 6-month relationship because mothers were our primary informants), and (e) obtain a Full-Scale IQ (FSIQ) between 50 and 90 on the Wechsler Preschool and Primary Scale of Intelligence-RevisedWPPSI-R (Wechsler, 1989).

A number of exclusionary criteria also were established. Based on the Child Behavior Checklist-CBCL (Achenbach, 1991) completed by the mother (or other female caregiver) for each child (see below), children who scored in the clinical range were excluded from the study (a $\mathrm{T}$ score above 70 was established for children with developmental delays to adjust to items focusing on developmental functioning). This rarely occurred because a phone screening interview for mothers eliminated children described as exhibiting major behavior problems. Similarly, exclusion occurred if mothers scored at or above the 95th percentile on the Parent domain of the Parenting Stress Index-PSI (Abidin, 1995). Finally, children were excluded if English was not their primary language or if they had significant sensory or motor problems.

Although children were selected strictly on the basis of developmental characteristics, we also obtained information with respect to child diagnosis. Because the diagnostic status of children with mild developmental delays is subject to change over time, parents provided information at the end of the study. Most children (81\%) received only categorical diagnoses (e.g., static encephalopathy, developmental delay) or no diagnosis whatsoever, with meaningful etiologic diagnoses infrequently reported. This pattern was similar for the two groups.

\section{Child and Family Measures}

We obtained numerous measures of child cognition and language, children's adaptive behavior, behavior problems, and social competence as rated by mothers as well as child behavior and social competence as rated by teachers. Self-report parent measures related to family demographics, stress, and support were also collected. Key mea- sures, including child CA, gender, and grade in school, are presented in Table 1.

Specifically, the WPPSI-R was administered to assess children's intellectual level. Older children were assessed with the Wechsler Intelligence Scale for Children-Third Edition-WISC-III (Wechsler, 1991). To provide an estimate of children's adaptive behavior, we administered the Vineland Adaptive Behavior Scales (Sparrow, Balla, \& Cicchetti, 1984) Survey Form to each mother (or primary female caregiver, but hereafter referred to only as mother) by trained interviewers. Standard scores were obtained for each of the four domains (Communication, Daily Living Skills, Socialization, and Motor Skills) as well as for the total adaptive behavior score. Only the total score was used for this analysis. The alpha coefficient for this study, averaged across the four domains, was .83 .

Mothers also assessed their child's behavior problems based on the CBCL. Mothers rated the frequency of different behavior problems from a 118-item questionnaire using a 3-point scale. Only the broad band Internalizing and Externalizing scales ( $\mathrm{T}$ scores), in conjunction with a total behavior problem score (see Table 1), were used for analysis. Higher scores indicate greater perceived behavior problems. Alpha coefficients for this study were .70 for Internalizing and .85 for Externalizing.

The Teacher's Report Form (Achenbach, 1991) was completed by each child's head teacher (separately for Year 1 and Year 2 in January and May, respectively). The Teacher's Report Form is designed to obtain standardized reports of children's behavioral/emotional problems that are similar to the CBCL as well as provide estimates of academic performance and adaptive functioning. This measure has 118 problem items, of which 93 have counterparts on the CBCL. Teachers were asked to rate the child with respect to how true each item is now or within the past 2 months using the same 3-point scale as found on the CBCL. Similar to the CBCL, only internalizing, externalizing, and total behavior problem scores (see Table 1) were used for analysis. Alpha coefficients for this study were .87 for internalizing and .93 for externalizing.

To obtain overall estimates of parenting stress, the PSI was administered to mothers. This instrument is a 101-item questionnaire that yields scores for two domains that differentiate sources of stress with good discriminant validity (Bigras, 
Table 1. Child and Family Measures by Groups at Pretest

\begin{tabular}{|c|c|c|c|c|}
\hline \multirow[b]{2}{*}{ Measure } & \multicolumn{2}{|c|}{ Intervention $(n=46)$} & \multicolumn{2}{|c|}{ Control $(n=44)$} \\
\hline & Mean/\% & $S D$ & Mean/\% & $S D$ \\
\hline \multicolumn{5}{|l|}{ Child demographics } \\
\hline Age at playgroup (months) & 63.57 & 7.40 & 64.17 & 8.36 \\
\hline Gender ( $\%$ male $)$ & 73.9 & & 75.0 & \\
\hline Grade in school (\% preschool) & 54.3 & & 40.9 & \\
\hline \multicolumn{5}{|l|}{ Child developmental } \\
\hline Full Scale IQ ${ }^{a}$ & 72.02 & 12.44 & 72.39 & 11.63 \\
\hline Vineland & & & & \\
\hline Adaptive Behavior/Total ${ }^{\mathrm{b}}$ & 70.78 & 10.05 & 73.09 & 10.64 \\
\hline \multicolumn{5}{|l|}{ Child behavior } \\
\hline $\mathrm{CBCL}$ total behavior problems ${ }^{c}$ & 58.22 & 6.65 & 58.77 & 7.63 \\
\hline TRF total behavior problems ${ }^{d}$ & 59.95 & 7.65 & 56.97 & 5.86 \\
\hline \multicolumn{5}{|l|}{ Parent stress and support } \\
\hline PSI Parent domaine & 126.07 & 23.29 & 120.30 & 26.34 \\
\hline \multicolumn{5}{|l|}{ Family demographics } \\
\hline Social status ${ }^{f}$ & 50.23 & 14.24 & 50.22 & 13.60 \\
\hline
\end{tabular}

Note. $N$ varies slightly for teacher report measure. Data for additional measures used to establish equivalence between groups is available from the first author.

a Weschler Preschool and Primary Scale of Intelligence-Revised. bVineland Adaptive Behavior Scales, standard scores.

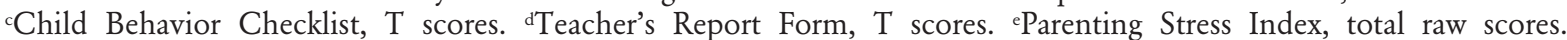
fHollingshead Four-Factor Index of Social Status.

LaFreniere, \& Dumas, 1996). The Parent domain $(\alpha=.89$; test-retest $=.81$ from standardization sample) is focused on mothers' reactions to the experience of being a parent and is composed of subscales referred to as Depression, Attachment, Restriction of Role, Sense of Competence, Social Isolation, Relationship With Spouse, and Health. This domain, summing across the seven subscales, represents perceived adverse impact on maternal functioning that may affect their parenting role and general well-being. The total raw score for the Parent domain was used for analysis. The alpha coefficient in this study was .85 for this domain.

Finally, standard demographic information about the family (marital status, number of children, ethnicity, educational and occupational status, and income) was gathered via self-reports from mothers. We used the Hollingshead Four Factor Index of Social Status (Hollingshead, 1975) to calculate a measure of family status (range $=8$ to 66 ).

\section{Experimental Design and Procedure}

Following recruitment procedures outlined above, children's cognitive and language devel- opment were evaluated subsequent to receiving all consent forms. Families whose children met all inclusionary criteria then received a packet of materials in the mail containing the study's scales and questionnaires. At the same time, mothers were scheduled to bring their child to the laboratory for pretest observations of child-child interactions (see below). A similar process of sending materials and scheduling was followed for the posttest. All interviews were administered to mothers during the laboratory visits. Teachers completed forms during the school year.

Based on pretest scores of children who were not excluded (see exclusionary criteria noted earlier; only 4 children were disqualified due to high CBCL or PSI scores), children were randomly assigned to control or intervention groups. Although no formal stratification procedure was applied, effort was made to ensure overall group equivalence by grouping children within cohorts based primarily on FSIQ scores (high/low median split) and then making random assignments. Even after attrition (see below), the procedure resulted in two groups similar on all measures presented 
in Table 1, and none of the $t$ test or chi-square analyses were significant at the .05 level.

Additional measures were also obtained to help establish equivalence between the groups. These included major subscales of the measures noted above as well as the following: the Test for Auditory Comprehension of Language-RevisedTACL-R (Carrow-Woolfolk, 1985); the Expressive One Word Picture Vocabulary Test-Revised (hereafter One Word Vocabulary Test) (Gardner, 1990); the parent form of the Social Skills Rating System (Gresham \& Elliott, 1990); the California Preschool Social Competency Scale-Teacher Completed (Levine, Elzey, \& Lewis, 1969); the Child domain of the PSI, and an index of mothers' social support based on the Inventory of $\mathrm{Pa}$ rental Experiences (Crnic, Greenberg, Ragozin, Robinson, \& Basham, 1983). As was the case for measures listed in Table 1, comparisons between groups did not indicate any significant differences. (Values for all measures for each group can be obtained by contacting the first author.)

Attrition was low throughout the course of the study, with only 6 families in the intervention and 9 families in the control group failing to complete the 2-year study period. Six families moved out of the area and 2 obtained jobs, making it difficult for them to participate. Reasons for dropping out varied for the other families (e.g., death of one mother, mothers indicated they were too busy to complete participation). Chi-square analyses showed no significant difference between intervention and control groups on the number of drops. In addition, a series of Condition (intervention vs. control) $\times$ Attrition (drop vs. nondrop) ANOVAs were carried out for all nondichotomous measures noted above. No significant main or interaction effects were obtained. Chisquare analyses for dichotomous variables also failed to produce significant differences for attrition group or condition. This yielded a final total of 90 participants $(n=46$ intervention; $n=44$ control).

\section{Laboratory Observations of Child-Child Interactions}

During the fall of the first year and the summer of the second year, children were brought to the laboratory for a series of observations of child-child interactions. Observations of focal children's play interactions with typically developing peers were carried out within a laboratory playroom designed to be similar to a typical preschool classroom. All observations were obtained at both pre- and postassessment periods for control and intervention groups. To record play interactions, we equipped the room with two video cameras operated by remote control, a radio telemetry microphone for the focal child, and an overhead microphone. A control panel with mixers balanced the auditory signals, allowing use of split screen technology for video input. Childchild interactions were observed in playgroups (quartets) with unfamiliar, typically developing peers (see discussion below).

We recruited typically developing children for the playgroups by distributing study announcements to local preschools, daycare centers, and schools. A brief telephone screen of interested parents was conducted during which the inclusion criteria for the children were described. Specifically, at pretesting typically developing children were required to be between the ages of 48 and 78 months at the time of the playgroups (same as the focal children); have no known developmental, sensory, motor, or behavioral problems; and have English as a primary language. To assure that the children were typically developing, we administered the TACL-R and the One Word Vocabulary Test to each child. To participate in the playgroups, children needed to obtain a standard score of at least 90 on each test. Children whose raw scores were greater than two $S D$ s above the mean based on the norms for 6.5-year-old children were excluded to eliminate excessive developmental differences between the typically developing children and the children with developmental delays. Mothers of typically developing children also completed the CBCL for their child. Those children who obtained $\mathrm{T}$ scores above 66 were excluded from the sample. Typically developing children were assigned on an availability basis but matched for gender with the focal child. None of the typically developing children had any prior contact with the focal children. Average peer age at pretest for the intervention group was 56.61 months and for the control group, 56.68. These two groups did not differ significantly from one another. Mothers also provided information to allow us to calculate the Hollingshead measure of social status. No differences in child (language, behavior) or family characteristics were detected, and none were found for participants in the intervention and control groups.

Inclusion criteria for the typically developing 
children at posttesting were the same as at pretesting, except that children were required to be between the ages of 70 and 96 months at the time of the playgroups. To assure that the children were developing typically, the Communication domain of the Vineland was administered to mothers on the phone; mothers also completed the CBCL. To participate, children had to obtain a standard score of 90 or above on the Communication domain and a $\mathrm{T}$ score of 66 or below on the CBCL. For posttesting, a new group of typically developing children was recruited and again assigned on an availability basis but matched for gender with the focal child. None of the typically developing peers at posttesting had prior contact with the focal children. Mothers also provided information to derive a measure of family social status. Average age in months of peers for intervention $(M=82.19)$ and control $(M=82.93)$ groups did not differ. As in the pretest, no other child or family measure varied for the peers participating in the quartets for the two groups.

Playgroup observations. Based on this matching process (CA and gender), each of the focal children participated in three 1-hour sessions with 3 typically developing peers in our laboratory playroom during each time period. This allowed naturalistic peer group interactions to be evaluated as they emerged. Although all 4 children were unfamiliar with one another prior to the playgroups, the 3 typically developing children were introduced to one another and allowed to play together for 30 minutes 1 day prior to the first session. The purpose of this procedure was to further challenge the focal child by approximating a peer group entry task. The successive 1 -hour periods allowed the focal child opportunities to become integrated within the group and permitted detailed observations of those interactions.

Playgroups were supervised by an experienced teacher in early childhood education. Children participated in a number of activities within each 1-hour session typical of young children's programs, including circle time, music, and snack. During the 30-minute free-play period, the children had access to an extensive array of toys and equipment in the playroom. There were separate areas for housekeeping, blocks, puzzles, games, and manipulative toy play activities. During the free-play period, teacher interactions were limited to providing assistance to ensure the children's safety. Focal children were videotaped during the 30-minute free-play periods that took place during each of the three sessions in each time period.

\section{Child-Peer Play Coding Schemes}

The 30-minute playgroup observations were coded using two well-established schemes: (a) the Play Observation Scale and (b) the Individual Social Behavior Scale. Only the play interactions of the focal child were coded.

Play Observation Scale. A time code superimposed on each videotape in conjunction with a remotely controlled tape-stop device allowed observers to view tapes at 10 -s intervals. Coders recorded the quality of social participation and levels of cognitive play during each 10-s interval using the revised version of the Play Observation Scale (Rubin, 2001). Videotapes were sent to Dr. Rubin's laboratory at the University of Maryland, where highly trained staff members who were blind to children's group status coded all sessions.

The Play Observation Scale consists of $10 \mathrm{mu}$ tually exclusive and exhaustive categories. The first 3 were derived from Parten's (1932) social participation categories consisting of the following play classifications: (a) solitary (playing alone), (b) parallel (playing next to another child), and (c) group (playing with another child; a combination of Parten's associative and cooperative play categories). Nested within these three social participation categories are five measures of cognitive play based primarily on the work of Smilansky (1968): (a) functional (simple repetitive play), (b) exploratory (examining physical properties of objects), (c) constructive (learns to use materials, creates something), (d) dramatic (role-taking and pretend play), and (e) games with rules (child behaves in accordance with prearranged rules). If any 10-s interval is coded as either solitary, parallel, or group play, then one of the five cognitive play categories is also scored (an "occupied" category was also available if the category of cognitive play could not be determined). We analyzed summary scores for the major categories of solitary, parallel, and group play, summing over the nested cognitive play measures. The only exceptions to this were the dramatic play categories because these measures have special relevance to children's peerrelated social competence (Howes, 1988). Specifically, group-dramatic play is associated with higher levels of peer competence, whereas both the nonsocial play categories of solitary-dramatic and parallel-dramatic play are associated with more 
immature and inappropriate forms of play with peers (Rubin, 1982).

The seven remaining main categories consisted of the following: (a) unoccupied behavior (child not playing), (b) onlooker behavior (child watches other children but does not enter into play), (c) peer conversation (talking, questioning, and suggesting to other children but not playing), (d) transitional (moving from one activity to another), (e) adult-directed (any activity with an adult), (f) uncodeable, and (g) out of room. Variations of this scale have been applied effectively to children with disabilities similar to those in this study for playgroups (e.g., Guralnick et al., 1996a, 1996b). Evidence with respect to convergent and discriminant validity suggests that the Scale of Social Participation constitutes a useful index of a more general construct of peer competence (Provost \& LaFreniere, 1991).

As noted, the Play Observation Scale was coded by a group of independent raters at the University of Maryland. Training for such coding was extensive and initially took place utilizing playgroup tapes from a separate study. For prestudy reliability for the full variable matrix, including cognitive play categories nested within the social participation categories, all raters reached the minimum criterion required based on Cohen's Kappa (к) and achieved a mean of .77. After training was completed, we calculated interrater reliability on approximately $20 \%$ of randomly selected quartet sessions between pairs of coders and produced an overall Kappa of .70. Intercoder differences were resolved through review and discussion. Intraclass correlation coefficients were also calculated for the main Play Observation Scale measures (see below) to index interrater reliability and were high in all instances $(M=.91$, range $=.73$ to .99$)$.

Individual Social Behavior Scale. Each videotape was reviewed a second time to examine specific peer-related social behaviors of the focal child. For this purpose, we used the Individual Social Behavior Scale, based on the work of White and Watts (1973) and adapted in a manner similar to Doyle, Connolly, and Rivest (1980) and to Guralnick and Groom (1985, 1987a, 1987b). A version of this scale was applied successfully with preschool-age children who had mild delays and communication disorders and to typically developing children as they interacted in playgroups similar to those described in this study (Guralnick et al., 1996a, 1996b).

In this study, observers unaware of children's group status continuously recorded the occurrence of individual social behaviors defined by 25 categories. The following categories were designed to record social interactions of the focal child as directed to peers: (a) seeks attention of peer; (b) uses peer as a resource; (c) leads in peer activitiesdirect, positive, or neutral; (d) leads in peer activities-indirect, positive, or neutral; (e) leads in peer activities-direct, negative; (f) leads in peer activities-indirect, negative; (g) joins peer(s) in specific activity, (h) expresses hostility toward peer, (i) takes unoffered object, (j) defends property, and (k) seeks agreement from peer.

The 14 remaining categories are focused on the social behaviors of the focal child in response to directed activities of peers. Categories consisted of following the lead of a peer (four categories tied to direct/indirect and positive/neutral, negative dimensions), failing to follow the lead of a peer (four categories as above), responding and failing to respond to a peer's attempt to use the focal child as a resource (two categories), responding and failing to respond to a peer's attention seeking behavior (two categories), and responding and failing to respond when a peer sought agreement from the focal child (two categories).

Coders were free to review any segment of the tape as often as needed. The coding protocol was divided into 30-s intervals following the time codes superimposed on the tape. Although coding was continuous, these divisions provided a structure for the coding task and served as a framework for establishing reliability within the event-based system. Coders were considered to be in agreement if codes matched within a specified 10-s interval using the "best fit" matching method (Hollenbeck, 1978). (A reliability manual describing this method is available from the first author.) In addition to the 25 Individual Social Behavior Scale categories, a no interaction event was included to complete the possible options within each 30 -s interval.

Prior to coding, four coders were trained for a period of 10 to 12 weeks on the Individual Social Behavior Scale. For prestudy reliability, calculated in this manner, all coders achieved the minimum average criterion for individual social behaviors necessary for participation of 75\% (overall $\kappa=.70$ ) on two consecutive 30-min tapes. Mean reliabilities for playgroup observations carried out for individual social behaviors during the course of the study on $20 \%$ of the total yielded a $\kappa=$ of .73 (range $=.54$ to .83 ) and mean per- 
centage agreement of $81.7 \%$ (range $=73.6$ to 92.0). To minimize observer drift, we held weekly coding meetings, and interobserver disagreements were resolved by discussion and reviewing tapes.

\section{Intervention}

The intervention process was initiated after all pretest assessments were completed and implemented from December of the first year (following the assessments) throughout the remainder of that school year, portions of the summer period (for the family component), and most of the next school year. Consequently, the total duration of the actual intervention was approximately 18 months.

No constraints were placed on the teachers or parents of control group children with respect to seeking other help with concerns about their children. Periodic contact was maintained with control group parents so as to limit dropouts. Newsletters with general parenting advice, holiday greeting cards, and phone calls were made to the parents of control group children every 3- to 4months after the pretest session and continuing until the posttest session.

The intervention consisted of a comprehensive program involving both family and school components. Based on a consultant service delivery model, intervention project staff worked closely with teachers and mothers to establish a positive relationship, to gather information through various assessments, and to develop joint plans for intervention in the form of specific goals and objectives to address issues identified. Both school and family components were organized through the use of standard protocols that consisted of clinical assessments yielding individualized profiles and providing a decision framework for prioritizing and developing intervention objectives linked to the assessment. Both assessment and intervention protocols were piloted for a 2-year period prior to this study.

All family- and school-based interventions were coordinated by project staff. For the school intervention, a doctoral-level educational psychologist well-trained in the intervention model coordinated all school interventions with the teachers at the school. For the family intervention component, a doctoral-level developmental psychologist well-trained in family systems and the intervention model coordinated the interventions with the mothers and children in the home and community. Meetings for each component were scheduled every other week with mother and teacher $(9$ months for the school component each year, with the family component extending into the summer), generally lasting 1 hour. Telephone contact was also maintained as needed. Bi-weekly staff meetings were held to review progress (detailed clinical records were maintained for each family and school visit) to ensure consistency with the conceptual model and to coordinate home and school approaches.

The child-focused aspects of the intervention for both teachers and mothers were organized in terms of the conceptual model outlined in the introduction. In the assessment phase, we utilized the Assessment of Peer Relations (Guralnick, 1992) for teachers or a modification for parents, which was developed for this project. The Assessment of Peer Relations is a clinical tool designed to gather information with respect to the developmental patterns of children's peer interactions and to evaluate relevant social-information and emotion-regulation processes in relation to children's use of social strategies. Major segments of the assessment are carried out in the context of three social tasks (peer group entry, conflict resolution, and maintaining play). Mothers or teachers are asked to first observe children in specific situations and then complete rating scales and checklists. Completion of the Assessment of Peer Relations or the parent version is carried out with the intervention consultant and forms the basis for intervention plans.

Highly individualized interventions based on jointly developed plans were designed first to promote the involvement of children in peer interaction activities. Strategies employed in this involvement phase included pairing children with compatible peers, selecting toys and activities of high interest, creating circumstances to minimize conflicts, and providing needed support and guidance from teachers or mothers. This was followed by efforts to enhance children's peer-related social competence in the context of the three social tasks. In this enhancement phase, for the intervention approach we relied extensively on the use of scripts to provide structure for each of the social tasks. The general plan was to help children organize the salient events represented in each of the social tasks (referred to as nodes) (see Nelson, 1981; Nelson \& Gruendel, 1979; Seidman, Nelson, \& Gruendel, 1986; Slackman \& Nelson, 1984). This approach is described in detail in the Enhancement of Peer Competence manual by

(C) American Association on Mental Retardation 
Neville, Connor, and Guralnick (2001), emphasizing the design and application of scripts for each of the three social tasks to be carried out within a pretend play framework. For example, when the social-information processing section of the Assessment of Peer Relations revealed that a child failed to attend properly to the play activities of peers in a peer group entry situation and did not establish a frame of reference, a "spy" script was developed, with accompanying props in which appropriate attention to the activities of peers before approaching the group was a central feature. This script was carried out in various settings and playgroups, with variations and techniques applied to assist the child to incorporate (generalize) those social strategies into more natural peer group situations. Emotion-regulation issues were frequently addressed in the script as well if indicated in the Assessment of Peer Relations results, using mood-induction strategies (Brenner, 2000; Carr, McLaughlin, Giacobbe-Grieco, \& Smith, 2003; Lemerise \& Arsenio, 2000). Both teachers and mothers were provided with guidance to organize and implement scripted peer play activities on their own either during playdates (mothers) or during school play times (teachers). Children's participation in the involvement and enhancement phases varied with child characteristics and responsiveness to the intervention. The primary goal was to improve the appropriateness and effectiveness of children's social strategies in the context of social tasks.

In addition to child-focused interventions, a number of important family influences on children's peer-related social competence were addressed. The domains relevant to peer-related social competence were certain aspects of parentchild interactions, direct parent actions in the form of arranging and monitoring peer play experiences, maternal attitudes with respect to factors governing children's peer competence, and family risk associated with stress and support. Considerable information was available for each of these domains as part of the formal pretest assessment package, as was information regarding each child's developmental profile. This information was then summarized in accordance with specific protocols developed by project personnel to prepare for a series of subsequent assessmentoriented interviews between the consultant and the mother. These interviews took place in the home and provided an opportunity to introduce each of the four domains to the mother and ex- plain their relationship to children's peer competence. This structured interview employed prompts in the form of questions in specific areas of interest (e.g., arranging play dates, possible causes of peer competence [internal vs. external], the balance in parent-child interactions [control], emotion-regulation exchanges in parent-child play, specific types of stress, and forms of social support available and unavailable). Based on a manual developed for the project, the consultant identified areas of strength and concern culminating in a jointly agreed upon selection of objectives and intervention plans in each of the four general domains. As noted, the ultimate goal of each family objective selected was to improve the peer competence of children in the intervention group. Approaches varied but included increasing children's experiences with peers through providing strategies to organize and support playdates. Other efforts to enhance social support, reduce stress, or manage their child's behavior, were intended to enable mothers to both focus more on intervention activities and to be more effective.

\section{Treatment Fidelity and Integrity}

The consultant model was designed to maximize the intensity of the intervention by having mothers and teachers implement agreed upon activities between meetings with the consultant. During visits to school or home, techniques were demonstrated as needed. However, the model involved developing a partnership with mothers and teachers, thereby precluding any attempt to directly monitor or evaluate their behavior.

Consequently, treatment fidelity was determined by asking teachers and mothers to discuss at each meeting the extent to which they completed the activities agreed upon during the previous meeting. The following 4-point scale was completed by the consultant to evaluate the percentage of planned actions conducted: 1 , no attempt; 2 , less than $50 \% ; 3,50 \%$ or more; or 4 , $100 \%$. This information provided an index of intensity of the intervention but not the quality of the activities. In addition, the number of visits and time spent by the consultants with mothers and teachers was recorded.

An alternative perspective on treatment fidelity was obtained through exit interviews with teachers and mothers. This structured interview obtained information with respect to the feasibility of the intervention, perceived effectiveness, overall degree of effort applied to the intervention 
activities, and positive and negative features of the intervention process.

For consultants, the integrity of the treatment was maintained through reliance upon specific assessment instruments, manuals, and intervention protocols. Detailed clinical records for each teacher and family contact were also maintained and served as the basis for bi-weekly meetings with the principal investigator. This also ensured a substantial degree of consistency between the conceptual model and implementation of the intervention approach.

\section{Results}

\section{Intervention Characteristics: Type, Intensity,} and Fidelity

As described in the introduction, the intervention protocol was highly individualized based on information obtained from the various assessments. For families, the main types of intervention objectives implemented were categorized as follows with the percentage of families receiving each intervention type noted in parenthesis: arranging and monitoring playdates (78.3\%), facilitating play during involvement using the Parent version of the Assessment of Peer Relations $(56.5 \%)$, facilitating play during enhancement $(30.4 \%)$, promoting mother-child play in the context of emotion-regulation (10.9\%), enhancing child directed play $(37.0 \%)$, addressing behavior management issues (26.1\%), and facilitating social support (13\%). Intervention objectives agreed upon with teachers were either of the involvement or enhancement type. All children participated in involvement with objectives related to understanding social roles and social rules (shared understanding) being most prevalent $(82.6 \%)$. For enhancement objectives, $18.3 \%$ participated in peer group entry, $8.7 \%$ in conflict management, and $10.9 \%$ in maintaining play interventions.

Across the intervention period, the mean number of contacts with teachers was 27.5 , with a mean total of 15.97 hours. For mothers, the mean number of contacts was 17.97 , with a mean total contact of 16.99 hours. The lower number of contacts with mothers reflects difficulties in scheduling and the conflicting commitments of families. Consequently, the mean number of direct total contact hours by the intervention consultants was only 32.96 across the entire intervention period. Moreover, hours devoted to actual intervention protocols were about 20\% less due to the time required for assessment activities during contacts. We note that no association was found between a variety of indices of intervention intensity (e.g., total hours) and any outcome measure (see below). In considering this result, we had concerns about the accuracy of the intensity reported by parents (and teachers) as well as the relatively narrow range of known values (e.g., faceto-face hours).

As described earlier, mothers and teachers were encouraged to follow the intervention protocols agreed upon during direct contacts to enhance the focal child's interactions with peers. To obtain information on the extent to which this was carried out, mothers and teachers completed a fidelity rating during each visit by the consultant. Mean fidelity ratings on a scale of 1 (none) to $4(100 \%)$ with respect to carrying out planned activities was 2.79 for mothers and 3.13 for teachers, indicating a modest to good level of reported involvement in the intervention. In structured exit interviews teachers were asked whether (a) implementation was easily accomplished, (b) it related to their curriculum, (c) the focal child enjoyed and responded to the intervention protocol, (d) the peers participating enjoyed the activity, and (e) instances of generalization were observed in activities other than those planned or with different play partners. Positive responses predominated $(3=$ yes; $2=$ yes, but concerns; $1=$ negative response), with an overall mean of 2.75 ( $S D=$ .20). Similar ratings from mothers regarding their feelings about the intervention, its effectiveness in promoting their child's social skills, ease of implementation, and amount of effort required were also positive $(M=2.38, S D=.33)$.

\section{Playgroup Observations: Individual Social Behavior Scale}

Four composite scores were first created from the Individual Social Behavior Scale categories to represent the dimensions of directing social interaction to peers and being responsive to peers as well as characterizing interactions as positive or negative. These four composite scores and the Individual Social Behavior Scale categories included in those scores are as follows: (a) positive directed to peer: joins peer, leads peer direct-positive/neutral, leads peer indirect-positive/neutral, uses peer as resource, seeks attention of peer, and seeks agreement from peer, $\alpha=.82$; (b) negative directed 
to peer: expresses hostility, leads peer direct-negative, leads peer indirect-negative, and takes unoffered object, $\alpha=.70$; (c) responsive to peer positive: follows peer direct-positive/neutral, follows peer indirect-positive/neutral; responds to peer as resource, responds to peer's attention-seeking, and responds to peer's agreement seeking, $\alpha=.84$; and (d) nonresponsive to peer positive: fails to follow peer direct-positive/neutral, fails to follow peer indirect-positive/neutral, fails to respond to peer seeking focal child as resource, fails to respond to peer seeking attention, and fails to respond to peer seeking agreement, $\alpha=.74$. Two summary scores, total positive behaviors, $\alpha=.89$, and total negative behaviors, $\alpha=.87$, were also created. The total positive behaviors summary score was created by combining the positive directed toward and responsive to peer positive composites above. The total negative behaviors summary score was created by combining the negative directed to and nonresponsive to peer positive composites above plus the following categories: follow peer directnegative, follow peer indirect-negative, fail to follow peer direct-negative, fail to follow peer indirect-negative, and defends. In addition to these six scores, measures of the proportion of positives directed to peers, the proportion of positive responses to peers, and the proportion of positives (overall to total) were calculated and included in the analysis (see Table 2). Based on intraclass correlation coefficients, we obtained high levels of interrater reliability for all measures used in the analyses $(M=.97$, range $=.95$ to .98$)$.

A Condition (intervention, control) $\times$ Time (pretest, posttest) mixed model MANOVA was first carried out on normalized scores (log transformations) for the dependent measures. The following four Individual Social Behavior Scale nonoverlapping composite measures were included in this analysis: positive directed to peer, negative directed to peer, responsive to peer positive, and nonresponsive to peer positive. Results failed to detect any overall effects involving condition, although a significant multivariate main effect for time was obtained, $F(4,85)=4.97, p<.01$. Separate ANOVAs were then conducted for the remaining measures. These analyses again indicated no significant effects involving condition.

Significant time effects were obtained in these analyses, however, for the following measures: positive directed to peer-proportion, $F(1,87)=$ $5.68, p<.05$, responsive to peer positive-proportion, $F(1,86)=8.84, p<.01$, total positive behaviors, $F(1,88)=13.26, p<.001$, and total positive behaviors-proportion, $F(1,88)=13.27, p<$ .001. In addition, follow-up $t$ tests for the Time factor for the four measures included in the MANOVA also produced significant findings for positive directed to peer, $t(89)=3.21, p<.01$, and responsive to peer positive, $t(89)=3.45, p<$ .01 .

Accordingly, as indicated in Table 2, although

Table 2. Individual Social Behavior Scale Measures for Condition and Time

\begin{tabular}{|c|c|c|c|c|c|c|c|c|}
\hline \multirow[b]{3}{*}{ Measure } & \multicolumn{4}{|c|}{ Pretest } & \multicolumn{4}{|c|}{ Posttest } \\
\hline & \multicolumn{2}{|c|}{ Intervention } & \multicolumn{2}{|c|}{ Control } & \multicolumn{2}{|c|}{ Intervention } & \multicolumn{2}{|c|}{ Control } \\
\hline & Mean & $S D$ & Mean & $S D$ & Mean & $S D$ & Mean & $S D$ \\
\hline Positive directed to peer** & 52.61 & 42.67 & 53.02 & 52.39 & 68.87 & 47.56 & 64.61 & 47.79 \\
\hline Negative directed to peer & 18.74 & 16.43 & 18.84 & 18.45 & 21.17 & 18.34 & 18.34 & 16.59 \\
\hline Responsive to peer positive** & 25.22 & 17.94 & 26.00 & 26.39 & 35.76 & 21.12 & 33.57 & 23.79 \\
\hline Nonresponsive to peer positive & 19.89 & 14.40 & 18.41 & 16.17 & 21.70 & 17.30 & 21.89 & 15.00 \\
\hline Total positive behaviors*** & 77.83 & 54.33 & 79.02 & 71.91 & 104.63 & 64.62 & 98.18 & 62.20 \\
\hline Total negative behaviors & 60.04 & 40.30 & 55.50 & 39.04 & 66.70 & 46.61 & 64.98 & 40.76 \\
\hline Positive directed to peer-proportion* & .72 & .17 & .73 & .19 & .79 & .13 & .77 & .17 \\
\hline $\begin{array}{l}\text { Responsive to peer positive-propor- } \\
\text { tion** }\end{array}$ & .56 & .17 & .53 & .18 & .64 & .14 & .59 & .17 \\
\hline $\begin{array}{l}\text { Total positive behaviors-propor- } \\
\text { tion***}\end{array}$ & .55 & .15 & .54 & .14 & .63 & .13 & .59 & .16 \\
\hline
\end{tabular}

Note. $N s=46$ for the intervention group and 44 for the control group. Measures are summed over the three playgroup sessions at each time period.

Significant effects for time: ${ }^{*} p<.05 . * p<.01 . *{ }^{* *} p<.001$. 
no significant intervention effects were found over the approximately 20 -month period between preand posttesting, we obtained significant increases for virtually all of the positive measures of peer interactions based on the Individual Social Behavior Scale, whereas negative interactions with peers remained unchanged.

Moderator analysis. As noted in the introduction, level of children's cognitive development constitutes an important dimension likely to influence the impact of any intervention program. Following the recommendations by Kraemer, Wilson, Fairburn, and Agras (2002) with respect to the role of moderators in randomized clinical trials, we conducted analyses using the following pretest levels of IQ as the moderator: (a) children who obtained an FSIQ of 70 or less (i.e., children meeting the key criterion for being classified as having mental retardation) and (b) children with an FSIQ of 71 and above. For each of the IQ subgroups, we performed $t$ test or chi-square analyses to determine whether there were differences between intervention and control groups on any of the pretest measures, including demographics, child and parent measures, and the observational measures. For the low IQ group, no differences were detected. Similarly, for the high IQ group, no differences were detected except for the California Preschool Social Competency Scale-teacher measure, where the control group was higher than the intervention group $(M \mathrm{~s}=75.87$ and 70.57 , respectively), $t(42)=2.73, p<.05$.

For the moderator analysis, we carried out a Condition (intervention vs. control) $\times$ Time (pretest vs. posttest) $\times$ Moderator (high vs. low IQ groups) MANOVA for the four nonoverlapping composite measures, which yielded a significant Condition $\times$ Time $\times$ Moderator multivariate effect, $F(4,83)=2.65, p<.05$. This was followed by a series of Condition $\times$ Time $\times$ Moderator mixed design ANOVAs performed on the remaining five measures. Two of these measures yielded a significant Condition $\times$ Time $\times$ Moderator interaction: responsive to peer positive-proportion, $F(1,84)=6.70, p<.05$, and total negative behaviors, $F(1,86)=5.36, p<.05$. Planned contrasts were then used to test the Condition $\times$ Time interaction for each level of the moderator only for those significant measures (four nonoverlapping composites, responsive to peer positive-proportion, total negative behaviors). None of the effects were significant for the high IQ group, but two measures produced significant ef- fects for the low IQ group: nonresponsive to peer positive, $F(1,38)=5.02, p<.05$, and responsive to peer positive-proportion, $F(1,36)=4.29, p<$ .05 . Total negative behaviors approached significance, $F(1,38)=3.41, p<.07$, and were included in subsequent analyses. Specifically, for these three measures, $t$ tests were then used to examine pre-post differences for each condition within the low IQ subgroup. Findings revealed that low IQ children in the control group became more nonresponsive to the positive bids of peers over time, $t(19)=2.20, p<.05$, whereas no change was detected for children in the intervention group. For the proportion of responsiveness to peer positive measure, $t$ tests revealed that the low IQ children in the intervention group became more responsive to peers over time, $t(17)=4.91, p<$ .001 , but there was no change for the control group children. Finally, for the total negative behaviors measure, $t$ tests indicated that total negative behaviors increased over time for the control group, $t(19)=2.15, p<.05$, but did not change for children in the intervention group. Figure 1 illustrates these findings in terms of percentage change from pretest to posttest for the three measures based on overall means (see Panel A). Taken together, the results of each of these three measures, although interrelated, provided unique information and clearly indicate that low IQ children in the intervention group become proportionately more positive over time when interacting with peers, but that low IQ children in the control group become more negative over time.

\section{Playgroup Observations: Play Observation Scale}

A Condition $\times$ Time mixed model MANOVA was first carried out on the eight normalized Play Observation Scale measures (log transformations) listed in Table 3 (uncodeable not included). Similar to the Individual Social Behavior Scale analyses, this analysis yielded only a significant multivariate time effect, $F(8,81)=6.63, p<.001$. Table 3 presents the means and $S D$ s for each condition and time for each of the Play Observation Scale measures. We note that with the exception of dramatic play (see below), the nested cognitive play categories were not analyzed separately but were included as part of the total frequency children engaged in solitary, parallel, and group play.

Follow-up $t$ tests for the various measures yielded significant time effects for solitary, $t(89)=$

(C) American Association on Mental Retardation 


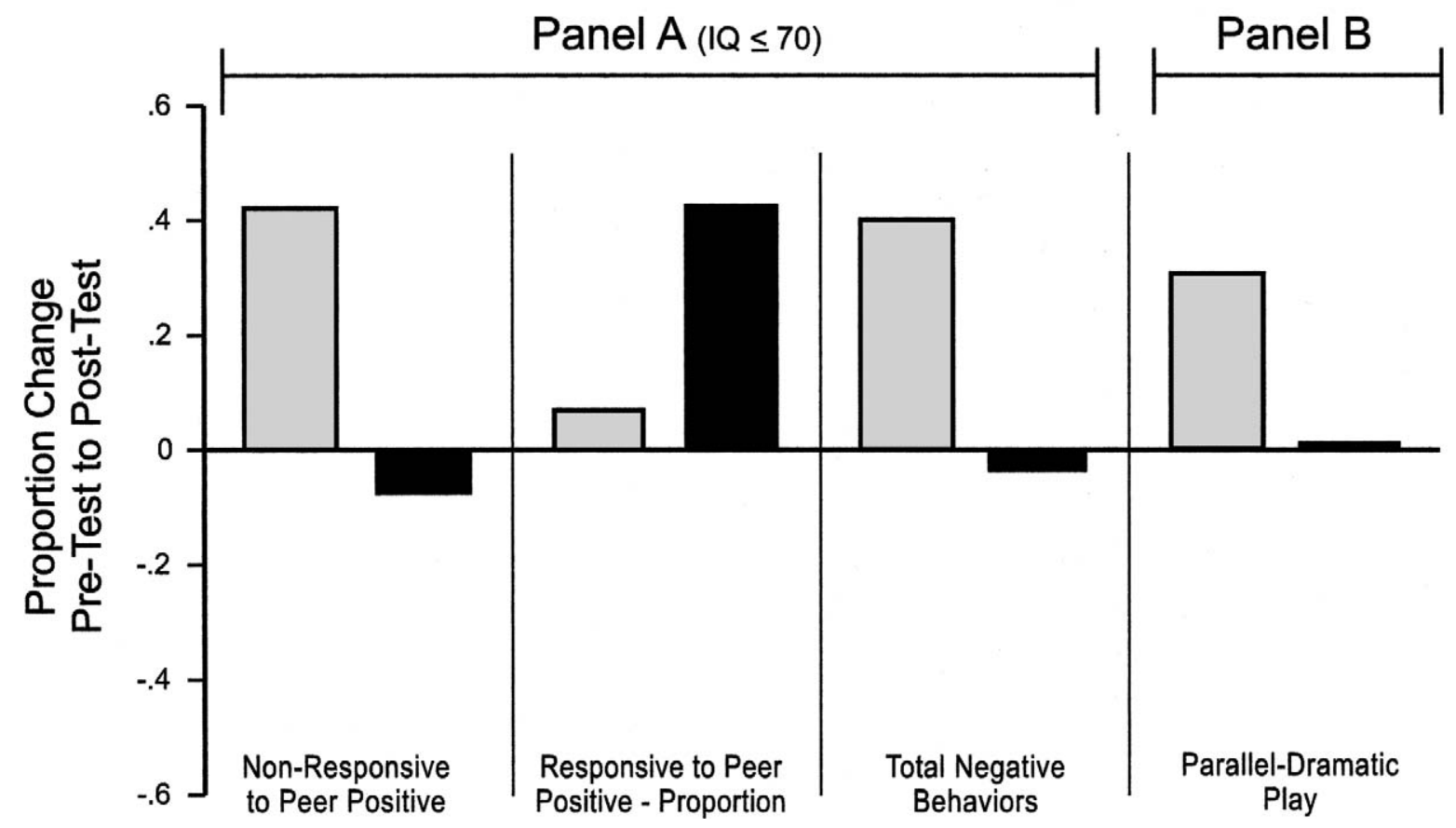

Figure 1. Proportion change from pretest to posttest by group for control and intervention groups. Panel A shows moderator effects for low IQ subgroup; Panel B, for overall effects for the Play Observation Scale parallel-dramatic play measure.

Table 3. Play Observation Scale Measures for Condition and Time

\begin{tabular}{|c|c|c|c|c|c|c|c|c|}
\hline \multirow[b]{3}{*}{ Measure } & \multicolumn{4}{|c|}{ Pretest } & \multicolumn{4}{|c|}{ Posttest } \\
\hline & \multicolumn{2}{|c|}{ Intervention } & \multicolumn{2}{|c|}{ Control } & \multicolumn{2}{|c|}{ Intervention } & \multicolumn{2}{|c|}{ Control } \\
\hline & Mean & $S D$ & Mean & $S D$ & Mean & $S D$ & Mean & $S D$ \\
\hline Solitary behaviors (Total)*** & 241.09 & 112.19 & 242.36 & 116.21 & 187.85 & 110.00 & 188.52 & 130.27 \\
\hline Parallel behaviors (Total)** & 92.43 & 48.49 & 79.09 & 57.04 & 107.52 & 53.75 & 100.27 & 61.26 \\
\hline Group behaviors (Total)*** & 20.20 & 25.04 & 24.02 & 30.45 & 35.93 & 51.11 & 47.14 & 53.81 \\
\hline Peer conversation** & 39.63 & 34.90 & 36.11 & 36.57 & 58.22 & 49.07 & 47.32 & 40.10 \\
\hline Adult interaction/conversation** & 7.48 & 7.54 & 7.25 & 6.08 & 5.89 & 6.60 & 4.70 & 4.77 \\
\hline Uncodeable & 9.20 & 10.71 & 12.34 & 13.52 & 7.96 & 9.73 & 12.14 & 14.62 \\
\hline Transitional & 35.61 & 17.29 & 32.36 & 13.86 & 29.65 & 16.91 & 32.32 & 19.04 \\
\hline Unoccupied & 22.96 & 13.11 & 31.98 & 25.46 & 25.09 & 18.82 & 22.18 & 15.87 \\
\hline Onlooker* & 60.70 & 48.78 & 57.30 & 54.76 & 70.67 & 53.46 & 71.66 & 58.93 \\
\hline
\end{tabular}

Note. $N s=46$ for the intervention group and 44 for the control group. Measures are summed over the three playgroup sessions at each time period.

Significant effects for time: $* p<.05 . * p<.01$. $* * p<.001$. 
4.66, $p<.001$, parallel, $t(89)=2.67, p<.01$, and group play, $t(89)=4.21, p<.001$. Significant time effects were also obtained for peer conversation, $t(89)=3.45, p<.01$; adult interaction, $t(89)=2.73, p<.01$; and onlooker behavior, $t(89)=2.62, p<.05$. Table 3 shows that children became more interactive with their peers over time as indicated by decreases in solitary play and interactions with an adult, but increases for all other measures from pretest to posttest periods.

In addition, as discussed earlier, because dramatic play is so critical in young children's peerrelated social interactions, we conducted separate ANOVAs for the nested solitary-dramatic, parallel-dramatic, and group-dramatic measures. A significant effect for the Condition $\times$ Time interaction was obtained for parallel-dramatic play, $F(1,88)=5.25, p<.05$. Follow-up $t$ tests indicated that intervention group children did not change their participation in this category over time (mean change from pretest to posttest was $+.11)$. However, control group children substantially increased participation in parallel-dramatic play over time, $t(43)=2.63, p<.05$ (mean change from pretest to posttest was 12.32) (see Panel B, Figure 1). No other effects were significant.

Moderator analysis. We then conducted a moderator analysis, similar to that carried out for Individual Social Behavior Scale measures, using the FSIQ cutoff of 70 for all measures in Table 3, except transitional, uncodeable, and adult interaction. An overall MANOVA showed no significant effects involving the Condition $\times$ Time $\times$ Moderator interaction. In addition, no significant effects involving the moderator variable were obtained for any of the three dramatic play measures.

In summary, for the Play Observation Scale measures, numerous changes occurred over time, indicating increasing involvement with peers similar to findings for the Individual Social Behavior Scale measures. The data also suggest greater involvement of control group children in paralleldramatic play from pretest to posttest but not for children in the intervention group. As discussed earlier, nonsocial forms of dramatic play are associated with maladaptive behavior and lower levels of social competence (Rubin, 1982). Of note, analysis of the Teacher's Report Form scores also revealed a significant correlation between the externalizing scale and parallel-dramatic play at pre- test and posttest, $r s=.29$ and .22 , respectively, $p s$ $<.05$.

\section{Discussion}

Establishing appropriate and effective interactions with peers constitutes a major concern for a substantial majority of young children with mild developmental delays. Early patterns of fragile, often negative, relationships with peers place these young children on a developmental trajectory destined to create long-term interpersonal problems and to diminish their quality of life. In the study reported here we addressed this critical issue in a randomized clinical trial evaluating the effectiveness of a comprehensive, developmentally oriented, and highly individualized intervention that extended over a 2-year period. Although the immediate impact of this intervention failed to produce extensive and large scale effects on children's competence with peers, the positive findings obtained were nevertheless highly encouraging. This is especially the case when it is recalled that, for this intervention to be considered successful, young children were required to display interactive competence under the most challenging of conditions (i.e., with unfamiliar peers in an unfamiliar setting).

Children in the low IQ subgroup (IQ $\leq 70$ ) benefited most from this intervention, reflected in three measures primarily related to negative interactions with peers. Whereas low IQ children in the control group showed increased negativity in their interactions over the 2-year period, no such changes were evident for the intervention subgroup. Over time, control group children in the low IQ subgroup became more nonresponsive to positive or neutral social bids by peers and increased the frequency of their total negative interactions with peers. Correspondingly, the proportion of positive responses to overall bids from peers did not change over time for the low IQ control subgroup but increased over time for the low IQ children who received the intervention. In addition, control group children increased their participation in the parallel-dramatic form of nonsocial play over time, but intervention group children's participation in this category remained stable. This finding was not related to children's cognitive level. Parallel-dramatic play suggests an inability to coordinate pretend play with other children and reflects a lack of social competence and lack of maturity (Rubin, 1982). In many ways, 
this form of play points to difficulties in shared understanding (i.e., knowledge of the social roles and social rules that guide well-defined pretend play scripts, Guralnick \& Neville, 1997). Our intervention did address many specific shared understanding objectives in the involvement phase of the curriculum, and there was a liberal use of scripts during the enhancement phase. Paralleldramatic play was also correlated with teacher reports of externalizing behavior, with items such as "argues a lot" or "stubborn, sullen, or irritable" contributing to that association. This pattern supports Rubin's (1982) suggestion that this form of nonsocial play may be associated with more aggressive or hostile play themes.

In terms of what was accomplished through this intervention, it cannot be said that children's peer-related social competence improved. Further evidence, particularly substantial increases in participation in group play, especially group-dramatic play, would be necessary for such a conclusion to be warranted. Perhaps the best way to characterize the positive findings of this study is to consider their potential preventive intervention value. By not allowing negativity to increase and by reducing the extent to which children engage in disconnected pretend play, often conflictual in nature, children are less likely to experience various forms of peer rejection over time (Coie, Dodge, \& Kupersmidt, 1990). Conflict resolution is clearly a major area of concern for children with developmental delays (Guralnick et al., 1998). Of note, evidence suggests that even more benign forms of nonsocial peer interactions, such as a preference for passive solitary activities, may well result in more difficult peer relationships over time (Rubin \& Mills, 1988). Whether intervention group children will ultimately be provided with more opportunities that enhance their peer-related social competence over time than children in the control group depends on many factors, particularly the type of supportive peer social environments in which children participate. It is worth noting that the primary impact on children's intellectual functioning as a result of comprehensive early intervention programs for children with cognitive delays has been to prevent declines in development from occurring (Guralnick, 1998). This is precisely the pattern obtained for the peer-related social domain in this study.

Future research designed to enhance the peer competence of young children with mild developmental delays should consider a number of is- sues that may have limited the effectiveness of the intervention in our work. Perhaps the most important factor was that our consultant model may have failed to generate a sufficiently high level of intensity to produce the desired effects. Despite an intervention protocol that spanned nearly 2 years, the total number of consultant hours and face-to-face intervention activities with mothers and teachers was rather small (less than 30 hours, on average, focusing on intervention itself). The intervention plan was designed to expand intervention intensity considerably through the participation of mothers and teachers. Nevertheless, despite efforts to comply, mothers, in particular, found it difficult to organize their schedule to carry out agreed upon activities. Teachers, too, often found it hard to find time to carry out intervention activities during the flow of the school day, despite exit interview information suggesting the feasibility of the activities and interest in participating. Discussions with teachers and occasional observations by consultants suggest that self-reported fidelity scores were likely overestimates of actual intervention activities.

The importance of intensity of intervention cannot be overemphasized, as this factor has emerged as perhaps the single most critical element in studies of the effectiveness of early intervention in general (Guralnick, 1998). For example, intervention intensity has been the key for both immediate and long-term effects for children born prematurely at low birth weight (Hill, BrookesGunn, \& Waldfogel, 2003; Ramey et al., 1992) and for children with autism (National Research Council, 2001). To achieve a sufficiently high level of intervention intensity in future studies to promote peer competence will likely require a substantially different implementation model than the one employed here. This is the case, despite the fact that this sample of families was clearly interested in promoting the peer-related social competence of their children, as judged by their willingness to volunteer for this study, and that most were two-parent families with adequate resources. Teachers seemed receptive to the program as well. Although the consultant model described in this study is not unusually expensive and does build partnerships with teachers and families, even with modifications to the model that bring in additional resources (e.g., involvement of teacher aides), dramatic increases in intervention intensity are unlikely to occur. Alternatively, consultants could work directly with 
teachers and children on a regular basis supplemented by small group intervention experiences for mothers and children. This model has been used by Webster-Stratton and her colleagues to successfully improve the behavioral development of young children from high-risk environments and those exhibiting conduct problems (WebsterStratton \& Reid, 2003; Webster-Stratton, Reid, \& Hammond, 2001).

As noted, children with low IQs benefitted more from the intervention than did children at higher cognitive levels. This outcome may be due to the fact that children at higher cognitive levels do not exhibit as severe problems in peer competence as do children at lower cognitive levels (Guralnick \& Groom, 1988; Guralnick et al., 1996b). In this study, children in the higher IQ subgroup interacted more extensively with peers than did children in the lower IQ subgroup based on the observational measures at pretest. Consequently, correspondingly small benefits of the intervention may not have been sufficient to produce significant effects for this higher functioning group. It is also possible that the more difficult problems exhibited by the lower IQ children may have focused the attention of mothers and teachers to a greater extent, perhaps realizing that these problems were not likely to diminish without concerted effort on their behalf (see Kopp et al., 1992). As such, mothers and teachers of low IQ children may have been more attentive and responsive to the intervention approach.

Relatedly, the range of the cognitive development of the children participating in the study was considerable, although variations across the dimension of cognitive level was useful in understanding the effects of this intervention. Nevertheless, future research should now consider more restrictive samples or ones that take into consideration co-occurring disabilities. For example, some children at the very high end of cognitive ability in our sample may have been able to meet criteria for specific language impairment (Leonard, 1998) or for a related communication disorder. These or other definable subgroups of children may exhibit more specific peer interaction and behavioral patterns over time and perhaps will display differential responsiveness to intervention as well (Benasich, Curtis, \&Tallal, 1993; Guralnick et al, 1996b; Guralnick, Hammond, \& Connor, 2006). Other children, including those at lower cognitive levels, may also have emerging co-occurring disabilities, such as various forms of per- vasive developmental disorder, that have a direct and substantial impact on social functioning (Klinger, Dawson, \& Renner, 2003).

Finally, it may be advisable for researchers in the future to work to directly evaluate the appropriateness and effectiveness of children's use of social strategies in social tasks related to peer group entry, conflict resolution, and maintaining play. This information, either obtained in playgroups or through more structured activities to elicit social strategies, would provide more specific insight into various aspects of children's peer-related social competence (Guralnick, 1999a) and serve as a useful complement to measures obtained in this study. Taken together, this information would permit future studies to refine intervention protocols even further.

In summary, this randomized clinical trial to promote the peer-related social competence of young children with mild developmental delays produced only modest results, with children with lower cognitive abilities benefiting most. Nevertheless, the benefits that did occur were obtained in highly challenging circumstances designed to probe generalization of peer interactions. As noted, generalization effects have been very difficult to achieve in this domain of development. The effects of this intervention are best conceptualized as preventing increases in children's negativity and unusual play patterns. Presumably, this will make it easier for children to develop more positive and more competent interactions with peers over time. In future work, programs must not only continue to address the child and family issues that formed the basis for this intervention, but must do so in a manner that emphasizes an implementation model that allows for a substantial increase in intervention intensity. Combined with greater attention to specific subgroups of children and families and other refinements in measurement systems, the lessons learned from the present investigation will, we hope, be useful in producing more effective intervention outcomes in this critical but neglected area of development for children with mild developmental delays.

\section{References}

Achenbach, T. M. (1991). Manual for the Child Behavior Checklist/4-18 and 1991 Profile. Burlington: University of Vermont, Department of Psychiatry.

American Psychiatric Association. (1994). Diag- 
nostic and statistical manual of mental disorders (4th ed.). Washington, DC: Author.

Bierman, K. L. (2004). Peer rejection. New York. Guilford Press.

Bigras, M., LaFreniere, P. J., \& Dumas, J. E. (1996). Discriminant validity of the Parent and Child scales of the Parenting Stress Index. Early Education and Development, 7, 167-178.

Booth, C. L. (1999). Beliefs about social skills among mothers of preschoolers with special needs. Early Education and Development, 10, 455-474.

Bray, N. W., Fletcher, K. L., \& Turner, L. A. (1997). Cognitive competencies and strategy use in individuals with mental retardation. In W. E. MacLean, Jr. (Ed.), Ellis' handbook of mental deficiency, psychological theory and research (3rd ed., pp. 197-217). Mahwah, NJ: Erlbaum.

Brenner, E. (2000). Mood induction in children: Methodological issues and clinical implications. Review of General Psychology, 4, 264-283.

Bryant, D., \& Maxwell, K. (1997). The effectiveness of early intervention for disadvantaged children. In M. J. Guralnick (Ed.), The effectiveness of early intervention (pp. 23-46). Baltimore: Brookes.

Carr, E. G., McLaughlin, D. M., Giacobbe-Grieco, T., \& Smith, C. E. (2003). Using mood ratings and mood induction in assessment and intervention for severe problem behavior. American Journal on Mental Retardation, 108, 32-55.

Carrow-Woolfolk, E. (1985). Manual for the Test for Auditory Comprehension of Language-Revised. Allen, TX: DLM Teaching Resources.

Coie, J. D., Dodge, K. A., \& Kupersmidt, J. (1990). Peer group behavior and social status. In S. R. Asher \& J. D. Coie (Eds.), Peer rejection in childhood (pp. 17-59). New York: Cambridge University Press.

Crnic, K. A., Greenberg, M. T., Ragozin, A. S., Robinson, N. M., \& Basham, R. B. (1983). Effects of stress and social support on mothers and premature and full-term infants. Child Development, 54, 209-217.

Denman, S. A., Blair, K., DeMulder, E., Levitas, J., Sawyer, K., Auerbach-Major, S., \& Queenan, P. (2003). Preschool emotional competence: Pathway to social competence? Child Development, 74, 238-256.

Dodge, K. A. (1991). Emotion and social information processing. In J. Garber \& K. A.
Dodge (Eds.), The development of emotion regulation and dysregulation (pp. 159-181). Cambridge: Cambridge University Press.

Doyle, A., Connolly, J., \& Rivest, L. (1980). The effect of playmate familiarity on the social interactions of young children. Child Development, 51, 217-223.

File, N. (1994). Children's play, teacher-child interactions, and teacher beliefs in integrated early childhood programs. Early Childhood Research Quarterly, 9, 223-240.

Gresham, F. M., \& Elliott, S. N. (1990). Social Skills Rating System. Circle Pines, MN: American Guidance Service.

Gresham, F. M., Sugai, G., \& Horner, R. H. (2001). Interpreting outcomes of social skills training for students with high-incidence disabilities. Exceptional Children, 67, 331-344.

Guralnick, M. J. (1992). A hierarchical model for understanding children's peer-related social competence. In S. L. Odom, S. R. McConnell, \& M. A. McEvoy (Eds.), Social competence of young children with disabilities: Issues and strategies for intervention (pp. 37-64). Baltimore: Brookes.

Guralnick, M. J. (1997). The peer social networks of young boys with developmental delays. American Journal on Mental Retardation, 101, 595-612.

Guralnick, M. J. (1998). The effectiveness of early intervention for vulnerable children: A developmental perspective. American Journal on Mental Retardation, 102, 319-345.

Guralnick, M. J. (1999a). Family and child influences on the peer-related social competence of young children with developmental delays. Mental Retardation and Developmental Disabilities Research Reviewes, 5, 21-29.

Guralnick, M. J. (1999b). The nature and meaning of social integration for young children with mild developmental delays in inclusive settings. Journal of Early Intervention, 22, 70-86.

Guralnick, M. J. (2001). Social competence with peers and early childhood inclusion: Need for alternative approaches. In M. J. Guralnick (Ed.), Early childhood inclusion: Focus on change (pp. 481-502). Baltimore: Brookes.

Guralnick, M. J. (2006). Family influences on early development: Integrating the science of normative development, risk and disability, and intervention. In K. McCartney \& D. Phillips (Eds.), Blackwell handbook of early childhood 
development (pp. 44-61). Oxford, UK: Blackwell.

Guralnick, M. J., Connor, R., Hammond, M., Gottman, J. M., \& Kinnish, K. (1996a). Immediate effects of mainstreamed settings on the social interactions and social integration of preschool children. American Journal on Mental Retardation, 100, 359-377.

Guralnick, M. J., Connor, R., Hammond, M., Gottman, J. M., \& Kinnish K. (1996b). The peer relations of preschool children with communication disorders. Child Development, 67, 471-489.

Guralnick, M. J., Gottman, J. M., \& Hammond, M. A. (1996). Effects of social setting on the friendship formation of young children differing in developmental status. Journal of $A p$ plied Developmental Psychology, 17, 625-651.

Guralnick, M. J., \& Groom, J. M. (1985). Correlates of peer-related social competence of developmentally delayed preschool children. American Journal of Mental Deficiency, 90, 140150.

Guralnick, M. J., \& Groom, J. M. (1987a). Dyadic peer interactions of mildly delayed and nonhandicapped preschool children. American Journal of Mental Deficiency, 92, 178-193.

Guralnick, M. J., \& Groom, J. M. (1987b). The peer relations of mildly delayed and nonhandicapped preschool children in mainstreamed playgroups. Child Development, 58, 1556-1572.

Guralnick, M. J., \& Groom, J. M. (1988). Friendships of preschool children in mainstreamed playgroups. Developmental Psychology, 24, 595604.

Guralnick, M. J., \& Groom, J. M. (1990). The relationship between parent-rated behavior problems and peer relations in preschool children. Early Education and Development, 1, 266278.

Guralnick, M. J., Hammond, M. A., \& Connor, R. T. (2006). Nonsocial play patterns of young children with communication disorders: Implications for behavioral adaptation. Early Education and Development, 17, 203-228.

Guralnick, M. J., \& Neville, B. (1997). Designing early intervention programs to promote children's social competence. In M. J. Guralnick (Ed.), The effectiveness of early intervention (pp. 579-610). Baltimore: Brookes.

Guralnick, M. J., Neville, B., Connor, R. T., \& Hammond, M. A. (2003). Family factors as- sociated with the peer social competence of young children with mild delays. American Journal on Mental Retardation, 108, 272-287.

Guralnick, M. J., \& Paul-Brown, D. (1989). Peerrelated communicative competence of preschool children: Developmental and adaptive characteristics. Journal of Speech and Hearing Research, 32, 930-943.

Guralnick, M. J., Paul-Brown, D., Groom, J. M., Booth, C. L., Hammond, M. A., Tupper, D. B., \& Gelenter, A. (1998). Conflict resolution patterns of preschool children with and without developmental delays in heterogeneous playgroups. Early Education and Development, 9, 49-77.

Guralnick, M. J., \& Weinhouse, E. M. (1984). Peer-related social interactions of developmentally delayed young children: Development and characteristics. Developmental Psychology, 20, 815-827.

Hill, J. L., Brooks-Gunn, J., \& Waldfogel, J. (2003). Sustained effects of high participation in an early intervention for low-birth-weight premature infants. Developmental Psychology, 39, 730-744.

Hollenbeck, A. R. (1978). Problems of reliability in observational research. In G. P. Sackett (Ed.), Observing behavior: Vol. 2. Data collection and analysis methods (pp. 79-98). Baltimore: University Park Press.

Hollingshead, A. B. (1975). Four factor index of social status. Unpublished manuscript, Yale University, Department of Sociology, New Haven.

Howell, A., Hauser-Cram, P., \& Warfield, M. E. (2001). Preschool predictors of later peer acceptance and loneliness in children with disabilities. Paper presented at the biennial meeting of the Society for Research in Child Development, Minneapolis.

Howes, C. (1988). Peer interaction of young children. Monographs of the Society for Research in Child Development, 53(1, Serial No. 217).

Jenkins, J. R., Odom, S. L., \& Speltz, M. L. (1989). Effects of social integration on preschool children with handicaps. Exceptional Children, 55, 420-428.

Kopp, C. B., Baker, B. I., \& Brown, K. W. (1992). Social skills and their correlates: Preschoolers with developmental delays. American Journal on Mental Retardation, 96, 357-366.

Kraemer, H. C., Wilson, T., Fairburn, C. G., \& Agras, W. S. (2002). Mediators and modera-

(C) American Association on Mental Retardation 
tors of treatment effects in randomized clinical trials. Archives of General Psychiatry, 59, 877-883.

Ladd, G. W., \& Pettit, G. S. (2002). Parenting and the development of children's peer relationships. In M. H. Bornstein (Ed.), Handbook of parenting: Vol. 5. Practical issues in parenting (2nd ed., pp. 269-304). Mahwah, NJ: Erlbaum.

Levine, S., Elzey, F. F., \& Lewis, M. (1969). California Preschool Social Competence Scale. Palo Alto, CA: Consulting Psychologists Press.

McConnell, S. R., McEvoy, M. A., \& Odom, S. L. (1992). Implementation of social competence interventions in early childhood special education classes: Current practices and future directions. In S. L. Odom, S. R. McConnell, \& M. A. McEvoy (Eds.), Social competence of young children with disabilities: Issues and strategies for intervention (pp. 277-306). Baltimore: Brookes.

McEvoy, M. A., Odom, S. L., \& McConnell, S. R. (1992). Peer social competence intervention for young children with disabilities. In S. L. Odom, S. R. McConnell, \& M. A. McEvoy (Eds.), Social competence of young children with disabilities: Issues and strategies for intervention (pp. 113-133). Baltimore: Brookes.

Michnowicz, L. L., McConnell, S. R., Peterson, C. A., \& Odom, S. L. (1995). Social goals and objectives of preschool IEPs: A content analysis. Journal of Early Intervention, 19, 273-282.

National Research Council. (2001). Educating children with autism. Committee on Educational Interventions for Children with Autism. Washington, DC: National Academy Press.

Nelson, K. (1981). Social cognition in a script framework. In J. H. Flavel \& L. Ross (Eds.), Social cognitive development: Frontiers and possible futures (pp. 335-364). Hillsdale, NJ: Erlbaum.

Nelson, K., \& Gruendel, J. (1979). At morning it's lunchtime: A scriptal view of children's dialogues. Discourse Processes, 2, 73-94.

Odom, S. L., McConnell, S. R., \& Chandler, L. K. (1993). Acceptability and feasibility of classroom-based social interaction interventions for young children with disabilities. $E x$ ceptional Children, 60, 226-236.

Odom, S. L., McConnell, S. R., McEvoy, M. A., Peterson, C., Ostrosky, M., Chandler, L. K., Spicuzza, R. J., Skellenger, A., Creighton, M., \& Favazza, P. C. (1999). Relative effects of interventions supporting the social competence of young children with disabilities. Topics in Early Childhood Special Education, 19, 7591.

Parten, M. B. (1932). Social participation among preschool children. Journal of Abnormal Social Psychology, 27, 243-269.

Provost, M. A., \& LaFreniere, P. J. (1991). Social participation and peer competence in preschool children: Evidence for discriminate and convergent validity. Child Study Journal, 21, 57-72.

Ramey, C. T., Bryant, D. M., Wasik, B. H., Sparling, J. J., Fendt, K. H., \& LaVange, L. M. (1992). Infant health and development program for low birth weight, premature infants: Program elements, family participation, and child intelligence. Pediatrics, 89, 454-465.

Rubin, K. H. (1982). Nonsocial play in preschoolers: Necessarily evil? Child Development, 53, 651-657.

Rubin, K. H. (2001). The Play Observation Scale. College Park: University of Maryland, The Center for Children, Relationships and Culture.

Rubin, K. H., Coplan, R. J., Fox, N. A., \& Calkins, S. D. (1995). Emotionality, emotional regulation, and preschoolers' social adaptation. Development and Psychopathology, 7, 4962.

Rubin, K. H., \& Mills, R. S. L. (1988). The many faces of social isolation in childhood. Journal of Consulting and Clinical Psychology, 56, 916924.

Seidman, S., Nelson, K., \& Gruendel, J. (1986). Make believe scripts: The transformation of ERs in fantasy. In K. Nelson (Ed.), Event knowledge: Structure and function in development (pp. 161-187). Hillsdale, NJ: Erlbaum.

Slackman, E., \& Nelson, K. (1984). Acquisition of an unfamiliar script in story form by young children. Child Development, 55, 329-340.

Smilansky, S. (1968). The effects of sociodramatic play on disadvantaged preschool children. New York: Wiley.

Sparrow, S. S., Balla, D. A., \& Cicchetti, D. V. (1984). Vineland Adaptive Behavior Scales. Circle Pines, MN: American Guidance Service.

Tomporowski, P. D., \& Tinsley, V. (1997). Attention in mentally retarded persons. In W. E. MacLean, Jr. (Ed.), Ellis' handbook of mental deficiency, psychological theory and research (3rd ed., pp. 219-244). Mahwah, NJ: Erlbaum. 
Webster-Stratton, C., \& Reid, M. J. (2003). Treating conduct problems and strengthening social and emotional competence on young children: The Dina Dinosaur Treatment Program. Journal of Emotional and Behavioral Disorders, 11(3), 130-143.

Webster-Stratton, C., Reid, M. J., \& Hammond, M. (2001). Preventing conduct problems, promoting social competence: A parent and teacher training partnership in Head Start. Journal of Clinical Child Psychology, 30, 283302.

Wechsler, D. (1989). Wechsler Preschool and Primary Scale of Intelligence-Revised. San Antonio: Psychological Corp.

Wechsler, D. (1991). Wechsler Intelligence Scale for Children (3rd ed.). San Antonio: Psychological Corp.

White, B. L., \& Watts, J. C. (1973). Experience and environment (Vol. 1). Englewood Cliffs, NJ: Prentice-Hall.

Williams, G. A., \& Asher, S. R. (1992). Assessment of loneliness at school among children with mild mental retardation. American Journal on Mental Retardation, 96, 373-385.
Wilson, B. (1999). Entry behavior and emotion regulation abilities of developmentally delayed boys. Developmental Psychology, 35, 214222.

Received 4/7/05, accepted 4/7/06.

Editor-in-charge: Frank Floyd

This research was supported by Grant RO1 HD37429 from the National Institute of Child Health and Human Development. Support from Grant P30 HD02274 also facilitated our work. The authors thank the many coders and other staff members at the University of Washington and the University of Maryland. We also are grateful to the families, teachers, and school district personnel for their participation in this effort to enhance the peer relationships of the vulnerable young children in their charge. Requests for reprints should be sent to Michael J. Guralnick, Center on Human Development and Disability, Box 357920, University of Washington, Seattle, WA 98195-7920. E-mail: mjgural@u.washington. edu 